\title{
Gender Differences in Stress among Parents of Children with Autism
}

\author{
Dr. Shahzadi Malhotra ${ }^{1}$, Prof. Waheeda Khan ${ }^{2}$, Prof. M.S. Bhatia ${ }^{3}$
}

\section{ABSTRACT}

Background: Researches in the recent years have consistently shown that parents of children with autism experience greater stress than parents of children without autism. There has been enormous understanding into the various challenges that these parents face. However, till date there have been no reported studies on gender differences in stress faced by parents of children with autism, especially in Indian context. Objective: The aim of the present research was to study and compare parenting stress and its domains in parents of children with autism. Participants and Methodology: The study sample consisted of 100 parents of children with autism- 50 mothers and 50 fathers selected using purposive sampling and fulfilling inclusion and exclusion criteria. Parenting Stress Index was used to study the total stress and its domains (child domain and parent domain) in the sample. Descriptive and inferential statistics was applied using SPSS version 17.0. Results showed no significant differences in total stress as well as on both the domains between the two study groups. To conclude, there are no gender differences in parenting stress among parents of children with autism.

Keywords: Autism, Parenting Stress

Autism is a neurodevelopmental disorder having early onset, and causes significant impairment in reciprocal social interaction and communication along with repetitive, stereotypical behaviours. Autism leads to increased care giving demands on the parents of children having this neurodevelopmental disorder. Parents of children with developmental disabilities including autism face numerous challenges. Further, because of the medical technological and health care advancements, lifespan of many children with disabilities has also increased causing further age related challenges for the parents. While providing care to a child is considered to be a normal part of being a parent of any child, providing higher levels of care necessary to a child with longterm functional limitations can become a strain and may result into impacts that affect the quality of life of the family caregivers, especially parents. Consequently, greater financial stress, more frequent disruption of family routine, and reduced social activities outside the family are basic problems that a family has to deal and cope with.

\footnotetext{
${ }^{1}$ Assistant Professor, Department of Clinical Psychology, Chacha Nehru Hospital, Delhi

${ }^{2}$ Department of Psychology, Jamia MIllia Islamia, New Delhi

${ }^{3}$ Head, Department of Psychiatry, GTB Hospital \& UCMS, New Delhi

*Corresponding Author

(C) 2015 I S Malhotra, W Khan, M Bhatia; licensee IJIP. This is an Open Access Research distributed under the terms of the Creative Commons Attribution License (http://creativecommons.org/licenses/by/2.0), which permits unrestricted use, distribution, and reproduction in any Medium, provided the original work is properly cited.
} 
Recently, researchers have shown that parents of children with disabilities face a lot more challenges and poorer psychological well-being than parents of children without any disability ${ }^{1}$.Having a child with a disability can impose financial, social and physical stress on the family ${ }^{2}$. Research has shown that caregivers of children with disabilities experience greater stress than caregivers of children without disabilities ${ }^{3}$. Similarly, Baker et al. ${ }^{4}$ have shown that parents of young children with developmental delays report experiencing greater stress than parents of children without delays. Thus, the presence of a child with developmental disability in the family involves continuous stress that incorporates many minor and major crises which call for a lot of adjustment for the family caregivers especially parents.

Despite so much understanding and research into the challenges that the family caregivers of children with various disabilities face, till date to the best of authors knowledge there have been no Indian studies on gender differences in stress of parents of children with autism.

Purpose: The purpose of the present research was to study and compare parenting stress and its dimensions in mothers and fathers of children with.

Design: A 2 group design (i.e. mothers of children with autism, fathers of children with autism) was used in the present study.

Sample: The study population consisted of children with autism; and their parents. Children with autism were those attending OPD at various neuro-psychiatric hospitals/ clinics and special schools in Delhi. Purposive sampling was used. The total sample of 100 consisted of 50 mothers and 50 fathers of children with autism.

\section{Inclusion Criteria for Children:}

1. Ages 5 years to 12 years.

2. Both genders.

3. Children diagnosed as cases of autism as per International Classification of DiseasesDiagnostic Criteria for Research (ICD-10-DCR).

\section{Exclusion Criteria for Children:}

Children with following comorbid conditions would be excluded from the study:

1. Epilepsy

2. Hearing impairment

3. Visual impairment

4. Motor impairment

5. Speech Impairment

6. Any other significant medical illness 


\section{Inclusion Criteria for Caregivers:}

1. Ages between 30 years to 45 years.

2. Minimum $12^{\text {th }}$ grade education

\section{Exclusion Criteria for Caregivers:}

1. Single parents

2. Caregivers having any significant medical/ psychiatric illness.

3. Any significant medical/ psychiatric family history that might contribute to caregiver burden.

Tools: The following tools were used in the study:

1. Sociodemographic Performa: It would consist of a structured format to record variables regarding the caregiver and the child with autism such as age and gender of child; age, gender, education, occupation, marital status of caregivers.

2. Childhood Autism Rating Scale (CARS) ${ }^{5}$ - The Childhood Autism Rating Scale (CARS) is a 15 item behavioural rating scale developed to diagnose and ascertain severity of autism.

3. Parenting Stress Index ${ }^{6}$ is a screening and diagnostic assessment tool designed to yield a measure of the magnitude of parent child system. It assesses stress experienced by the parent in the child domain, parent domain and the total stress. For the purpose of present research, the PSI was translated into Hindi using the back translation method.

There are 120 statements in the PSI form. These items are preceded by a cue to use a different response format. In addition, items 102 to 120 are Life stress (LS) items. The life stress scale is optional and has not been used in the present study. The raw scores are transformed into percentiles by child age. The following are the three dimensions assessed by PSI:

- Child Dimension assesses the child characteristics that may contribute to parental stress. It includes the child's level of distractability, adaptability, hyperactivity, child's mood and demandingness. It also assesses whether the child- parent interaction reinforces the parent.

- Parent Dimension of parenting stress assesses parent's level of isolation, depression, attachment with the child, health, role restriction (how much the parents experience their parenting role to be restricting their personal freedom), spousal support and competence.

- $\quad$ Total Stress is the combined score of child and parent dimension.

\section{PROCEDURE}

All children who were referred with a provisional diagnosis of autism were evaluated in the following way for the purpose of study: 
The inclusion and exclusion criteria were applied to the children and their caregivers and those who fulfilled the criteria were furnished with the necessary information about the study and a written informed consent was obtained from caregivers. Those who gave their consent were recruited and further evaluated for the study. Data was collected in the clinical setting. The nature of the study was explained to the caregivers and their consent taken. Further, sociodemographic Performa was administered to elicit information about sociodemographic, clinical and caregiver details. Diagnostic confirmation of children was done by administering CARS. After assessments with the children, Parenting Stress Index was administered on parents.

\section{RESULTS}

Data obtained has been analyzed using descriptive and inferential statistics. The Statistical Package for Social Sciences (SPSS, version 17.0) was used. Significance level $p<.05$ was regarded as statistically significant.

Out of the 64 families screened for inclusion and exclusion criteria, 14 did not meet the inclusion criteria and were therefore not included in the study. The mean age of the children was 10.9 $( \pm 1.8)$ years, the mean age of mothers was $38.6( \pm 3.44)$ years and the mean age of fathers was $42.8( \pm 4.01)$ years. The mean SQ of children was $48.42( \pm 12.83)$ and the mean CARS score was 40.5. Tables 1 show the summary of t-test of parenting stress and its dimensions between mothers and fathers of children with autism.

Table 1: Summary of t-test of dimensions of Parenting Stress of mothers and fathers having children with Autism (df =98)

\begin{tabular}{|c|c|c|c|c|c|c|c|c|}
\hline \multirow{2}{*}{ GS } & \multicolumn{3}{|c|}{ Mothers } & \multicolumn{3}{|c|}{ Fathers } & \multirow[b]{2}{*}{ t-value } & \multirow[b]{2}{*}{ p-value } \\
\hline & Mean & SD & SEM & Mean & SD & SEM & & \\
\hline $\begin{array}{l}\text { Child } \\
\text { Dimension }\end{array}$ & 83.99 & 15.86 & 2.24 & 78.10 & 17.83 & 2.52 & 1.74 & 0.0841 \\
\hline $\begin{array}{l}\text { Parent } \\
\text { Dimension }\end{array}$ & 86.01 & 16.95 & 2.37 & 80.84 & 15.39 & 2.18 & 1.60 & 0.1135 \\
\hline Total Stress & 85.99 & 16.16 & 2.34 & 80.26 & 16.08 & 2.27 & 1.76 & 0.0820 \\
\hline
\end{tabular}


From Table 1 it is observed that there were no statistically significant differences between mothers and fathers of children with autism on any of the two dimensions of parenting stress as well as total stress. Thus, the hypothesis is rejected and it is inferred that mothers and fathers of children with autism did not differ on the dimensions of parenting stress.

\section{DISCUSSION:}

There is considerable understanding that parents of children with developmental disabilities experience increased levels of stress, often related to the severity of their child's behaviour. However, the experience of stress is dependent on how individuals perceive their situation and what are the coping strategies (i.e., problem-focused, emotion focused, and appraisal- or perception-focused coping) that are used to manage stress ${ }^{7}$. Research has undoubtedly shown that caregivers of children with disabilities experience greater stress than caregivers of children without disabilities ${ }^{3}$. Similarly, Baker et al. ${ }^{4}$ have shown that parents of young children with developmental delays report experiencing greater stress than parents of children without delays. It has been suggested that the experience of stress is related to how the individual perceives the stressful event and whether coping strategies can be used effectively to manage stress ${ }^{2}$. However, to the best of authors' knowledge, there is no reported literature on the gender differences in parenting stress among parents of children with autism in Indian context. The present study was a step to fill this gap in the understanding of gender differences in parenting stress of parents having children with autism.

There are numerous effects like social and psychological impact on the lives of parents of children with disabilities. There is also evidence that high levels of stress are associated with the task of caring for a child having disability ${ }^{8}$, including autism9. From the results section, it is observed that there were no statistically significant differences on domains of parenting stress and total stress between the two study groups. Thus, although there are no statistically significant gender differences in parenting stress, there are differences in the way mothers and fathers perceive stress with regards to their own and child characteristics. This could probably be because both mothers and fathers face their own individual types of challenges and stressors when caring for their child with autism. For example, mothers may be overburdened by looking after the adaptive needs of their child while fathers may feel stressed owing to the overburden of making more money to meet the increased financial needs to look after the medical and rehabilitation expenses of the child with autism.

These findings are of concern as distress among parents has been linked to a wide range of adverse outcomes for children, including less than optimal functioning, failure to engage with services, decisions to seek out-of-home care for their disabled child, impeded child development, and higher rates of child psychopathology and antisocial behaviour ${ }^{10}$. The increased parenting stress is associated with lower levels of life satisfaction and marital satisfaction, poor parent-child communication and disruptive child behaviour which could have significant effects on mothers' physical and psychological well-being. Considering the results 
of the present study, both mothers as well as fathers need equal support and guidance to be equipped with such coping so as to enhance their physical and psychological well being.

To conclude, there are no significant gender differences in the parenting stress of mothers and fathers of children with autism. Both mothers and fathers have increased stress levels and future studies need to focus on interventions to lower this parenting stress.

\section{REFERENCES}

Achilles GM, McLaughlin MJ, \& Croninger RG. Sociocultural correlated of disciplinary exclusion among students with emotional, behavioural, and learning disabilities in the SEELS National Dataset. Journal of Emotional and Behavioural Disorders, 2007, 15(1), 33-45.

Mak WS, Ho SM. Caregiving perceptions of Chinese mothers of children with intellectual disability in Hong Kong. Journal of Applied Research in Intellectual Disabilities, 2007, 20, 145-156.

Hassall R, Rose J, McDonald J. Parenting stress in mothers of children with an intellectual disability: The effects of parental cognitions in relation to child characteristics and family support. Journal of Intellectual Disability Research,2005, 49, 405-418.

Baker BL, Blacher J, Crnic KA, Edelbrock C. Behavior Problems and Parenting Stress in Families of Three-Year-Old Children with and without Developmental Delays. American Journal of Mental Retardation, 2003, 107 (6), 433-444.

Schopler E, Reicher RJ \& Renner BR. The Childhood Autism Rating Scale (CARS). USA: Western Psychological Services 1980.

Abidin RR. Parenting Stress Index Professional Manual. $3^{\text {rd }}$ Edition.1995 North Florida, US: Psychological Assessment Resources.

Lopez V, Clifford T, Minnes P, Ouellette-Kuntz H. Parental stress and coping in families of children with and without developmental delays. Journal on Developmental Disabilities, 2008, 14(2), 99-104, Retrieved from http://www.oadd.org/Journal_14.html.

Salovita T, Italianna M, Leinonen E . Explaining the Parental Stress of Fathers and Mothers Caring for a Child with Intellectual Disability: A Double ABCX Model. Journal of Intellectual Disability Research,2003, 47 (4-5), 300-312.

Duarte C, Bordin I, Juliamooney L. Factors associated with stress in mothers of children with autism. Autism, 2005, 9(4), 416-427.

Llewellyn G, McConnell D, Thompson K, Whybrow S. Out-of-home placement of school-age children with disabilities and high support needs. Journal of Applied Research in Intellectual Disabilities,2005, 18(1), 1-6. 